\title{
On the use of the FBMC modulation to increase the performance of a wake-up radio
}

\author{
Florin Hutu and Guillaume Villemaud \\ florin-doru.hutu@insa-lyon.fr
}

Univ Lyon, INSA Lyon, Inria, CITI, F-69621 Villeurbanne, France

\begin{abstract}
In this paper, Filter Bank Multi Carrier (FBMC) signals are employed in order to improve the performance of a quasi-passive wake-up radio receiver (WuRx) for which the addressing is performed by the means of a frequency fingerprint. The feasibility of such kind of WuRx was already demonstrated by using orthogonal frequencydivision multiplexing (OFDM) signals to form the identifiers. Together with the main advantage of this approach (i.e. no base band processing needed and consequently a reduced energy consumption), one of the drawbacks is their low sensitivity. Through a set of circuit-system co-simulations, it is shown that by their characteristics, especially high Peak to Average Power Ratio (PAPR) and high out of band attenuation, FBMC signals manage to boost the sensitivity and moreover to enhance the robustness of this kind of WuRx.
\end{abstract}

Index Terms-5G mobile communication, energy efficiency, Internet of Things, OFDM, peak to average power ratio.

\section{INTRODUCTION}

By switching off the main radio transceiver during the inactivity periods, a WuRx manage to increase the overall energy efficiency of a connecting object. Indeed, knowing that, most of the time, the main radio front-end is activated without receiving data and it is one of the main energy consuming part of a connecting object, the gain in terms of energy efficiency may be important, especially in the case of low duty cycle transmissions.

A typical application scenario is the Internet of Things (IoT) where connecting objects with limited resources (computational, energy, etc.) are deployed. In the near future, billions of such connecting objects will be installed, will have the ability to transmit information about their environment and consequently to make an even stronger connection between the real and the digital worlds. Indeed, since the birth of the IoT (2008/2009), when the number of connected objects surpassed the world's population, today Gartner agency forecast is of 20.8 billion from 2020 and, for the same horizon, CISCO forecasts 50 billion [1]. On the same note, the IoT was identified as a potential target for the future $5 \mathrm{G}$ mobile communications standard [2] and the FBMC modulation is a candidate for the next generation of mobile networks [3].

Some active WuRx are existing, but requiring to demodulate some information in order to identify the ap- propriate radio interface to wake up, therefore inducing a higher energy consumption [4], [5], [6]. Another particular technique was proposed and experimentally validated by the authors in [7], [8]. The proposed solution is a tradeoff, enabling to identify the devices via a specific OFDM frequency pattern, conducing to a quasi-passive WuRx with no need of demodulation.

Efforts are undertaken to propose high performances wake-up radios while reducing the energy consumption. One of the ways to increase the sensitivity of the receivers is to use techniques resembling to the ones used in radio frequency (RF) energy harvesting and wireless power transfer, i.e. power optimized waveforms. In [9], the authors demonstrate that high PAPR signals are enhancing the efficiency of the rectifier circuits and in [10], the authors are using this results by proposing a dual band WuRx with increased performance.

Moreover, recent research is focusing on generating new waveforms in order to adapt the signal carrying the energy to the propagation channel [11]. The use of large-scale antenna arrays in order to increase the efficiency of wireless power transfer is intensively studied for $5 \mathrm{G}$ mobile communications standard since beamforming techniques may overcome the propagation channel effects.

In the same spirit, this paper presents the advantages of using the FBMC modulation technique in order to enhance the performances of the quasi-passive WuRx presented in detail in [7], [8]. The sensitivity, latency and the robustness related to the implementation impairments are compared to the OFDM case.

The rest of the paper is structured as follows: in the second section, the principle of the studied wake-up radio architecture and the main characteristics of the FBMC based identifiers will be described. In the third section, the simulation framework will be presented and the results demonstrating the efficiency of the FBMC signals will be highlighted. Finally, the last section will give the conclusions and the future directions of this work.

\section{USING FREQUENCY FINGERPRINTS AS IDENTIFIER SIGNALS}

One of the common ways to reduce the energy consumption of the connected objects and implicitly increase 
their autonomy is to propose energy friendly MAC (media access control) mechanisms consisting in turning off periodically the radio transceiver. This seems to be a good solution for the low duty cycle transmissions but it can lead to an increase of the network delay and to a less reliable communication, which contradicts the requirements of certain critical applications. Another way to reduce their energy consumption is to attach an auxiliary, ultra-low energy consumption receiver, called wake-up radio ( $\mathrm{WuRx}$ ). The role of a wake-up radio is to listen to the communication channel and to switch on the main transceiver only when a communication demand appears. Recent researches were able to decrease the energy consumption of the wakeup radios to levels below a microwatt [12], in such a way that it becomes feasible to keep them turned on permanently during the standby period. However, for low duty cycle scenarios, this standby energy consumption is still important.

Current technologies employed for the wake-up radio receivers varies from simple passive energy detector to more complex super-heterodyne active receivers. Indeed, some of WuRx have super-heterodyne or super regenerative structures and in these cases, the sensitivity is privileged at the expense of higher energy consumption. Other WuRx are based on injection locked oscillators or on envelope detectors that may be followed by IF (intermediate frequency) stages. In the latter cases, after the envelope detectors, a purely analog correlator circuit or a microcontroller performs the identification of the RF interface to wake up. Table I gathers some of the noticeable wake-up architectures.

\begin{tabular}{|c|c|c|c|}
\hline Reference & Frequency & DC Power supply & Sensitivity \\
\hline \hline$[4]$ & $868 \mathrm{MHz}$ & $40.9 \mu W$ & $-78 \mathrm{dBm}$ \\
\hline$[5]$ & $868 \mathrm{MHz}$ & $1.2 \mu W$ & $-55 \mathrm{dBm}$ \\
\hline$[6]$ & $113.5 \mathrm{M} \mathrm{Hz}$ & $4.5 \mathrm{nW}$ & $-69 \mathrm{dBm}$ \\
\hline$[10]$ & $868 \mathrm{MHz}$ & - & $-63 \mathrm{dBm}$ \\
& $2.45 \mathrm{GHz}$ & - & $-65 \mathrm{dBm}$ \\
\hline$[13]$ & $433 \mathrm{MHz}$ & $1.07 \mu W$ & $-51 \mathrm{dBm}$ \\
\hline$[14]$ & $868 \mathrm{MHz}$ & $2.4 \mu W$ & $-71 \mathrm{dBm}$ \\
\hline$[15]$ & $400 \mathrm{MHz}$ & $3.4 m W$ & $-80.2 \mathrm{dBm}$ \\
\hline$[16]$ & $2.4 \mathrm{GHz}$ & $2.59 \mathrm{~mW}$ & $-75 \mathrm{dBm}$ \\
\hline$[17]$ & $3.1-10 \mathrm{GHz}$ & $4.5 \mathrm{~mW}$ & - \\
\hline$[18]$ & $868 \mathrm{MHz}$ & $94.2 \mu W$ & - \\
\hline$[19]$ & $868 \mathrm{MHz}$ & $2.7 \mu W$ & - \\
\hline
\end{tabular}

TABLE I

NON-EXHAUSTIVE STATE OF THE ART OF WURX

The literature shows that, in most of the cases, the On-Off Keying (OOK) modulation is used in order to send the identifier of the main radio interface to wakeup. This modulation is preferred because the demodulator may be a simple rectifier circuit. Consequently, because no active frequency translation stage is employed, the energy consumption is drastically reduced. In order to reduce it even more, in [7], [8], the authors proposed another way to transmit the identifiers: by shaping an OFDM spectrum. More precisely, the 64 sub carriers of an $802.11 \mathrm{n}, 20 \mathrm{MHz}$ OFDM signal are grouped into $N=4$ subsets of sub carriers. By sending random data on a particular subset, the power spectral density on the corresponding $5 \mathrm{MHz}$ bandwidth is increasing and this is associated to a logical level 1. On the contrary, if no data is sent on the sub carrier set, the power level remains at a low level which is associated to a logical level $\mathbf{0}$. By using this binary alphabet, 14 possible identifiers may be built. As presented in section III, a quasi passive circuit, based on multi band filters is able to detect this kind of frequency pattern.

With the price to pay of the high computational complexity, compared to the classical OFDM modulation scheme, the FBMC modulation allows a better usage of the allocated spectrum and reduces the overall energy consumption. This is possible because in the latter case, the entire symbol period is used i.e. no cyclic prefix is needed. Also, in the case of the FBMC modulation, the out of band attenuation is increased and so, the neighbor users are protected. Moreover, the PAPR is increased which increases the efficiency of the rectifier circuits [20].

In FBMC, as in OFDM, the bitstream is divided in a number of substreams equal to the number of the subcarriers. These sub-carriers are orthogonal in order to overcome the inter symbol interference issues. A synthesis bank filter is used at the emission and a analysis bank filter at the reception. Generally, the filters are a set of bandpass filters which are frequency shifted or modulated versions of a prototype bandpass filters (cosine or exponential).

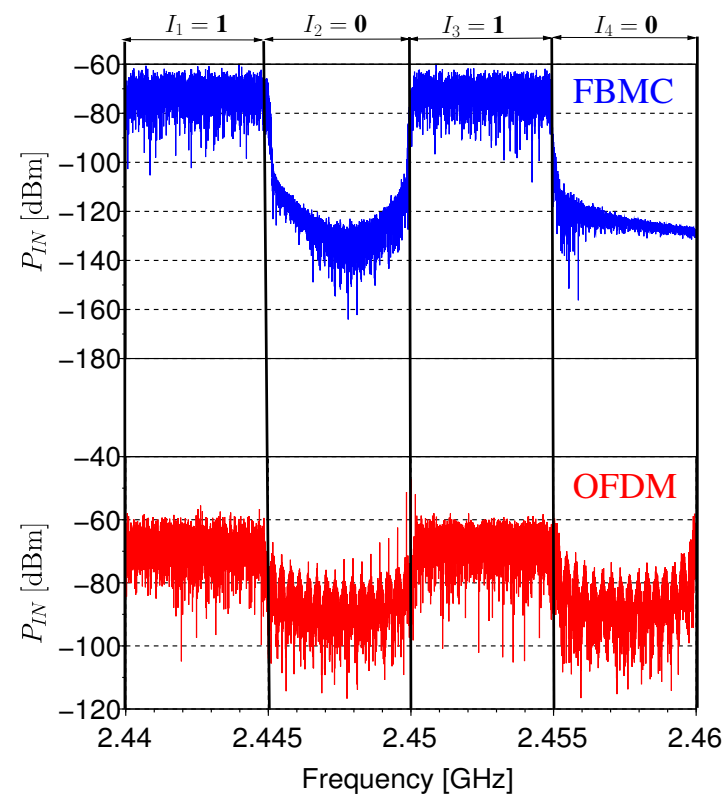

Fig. 1. A comparison between the FBMC and OFDM signals used as identifiers

In our case, in order to form the identifiers, in a $20 \mathrm{MHz}$ 
bandwidth, the 128 sub carriers of a FBMC signal were grouped into four sub bands. Each sub carrier forming a logical level 1 was modulated using random data and a QPSK modulation. In order to increase the out of band rejection, the FBMC baseband filters' overlapping factor (i.e. the number of multi carrier symbols that overlaps in time domain) was set to 4. As a comparison, in Figure 1 the FBMC and the OFDM versions of the $\mathbf{1 0 1 0}$ identifier are presented.

\section{Simulation Results}

In order to demonstrate the utility of using FBMC based identifiers, the simulation framework corresponding to the quasi-passive wake-up receiver, depicted in Figure 2, was implemented.

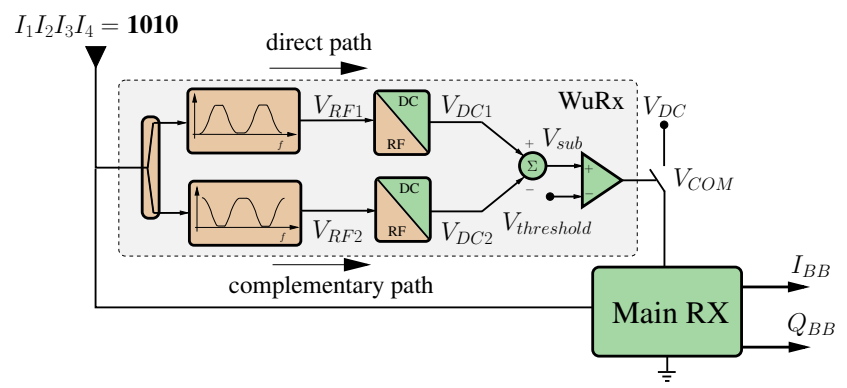

Fig. 2. The architecture of the quasi-passive WuRx driving the power supply of the main radio transceiver

The WuRx having the address $\mathbf{1 0 1 0}$ was simulated by using a set of band pass Chebyshev filters of order 15 having $1 \mathrm{~dB}$ band pass ripple, $-50 \mathrm{~dB}$ out of band attenuation, and $1 \mathrm{~dB}$ insertion loss. The rectifier circuits have been implemented by using HSMS 2850 Schottky diode's model. In order to maximize the power transfer between the filter banks outputs and the diode based rectifiers, a matching circuit was calculated by using lumped reactive components. The subtractor was implemented by using a classical op amp structure, which is inserting a gain of 100. The trigger level was arbitrarily set to $1 \mathrm{mV}$. The identifiers were formed by using OFDM and FBMC signals, as specified in the previous section and depicted in Figure 1.

As can be seen from Figure 3, compared to the OFDM case, the subtractor's output has a greater voltage in the case of using FBMC formed identifier. This can be explained by the higher PAPR of the FBMC signal that increases the efficiency of the diode based rectifier circuit. Consequently, the DC voltages at the outputs of the two rectifier circuits are greater than in the case of using OFDM signals. Thus, for the same threshold level, the activation of the wake-up radio occurs for power levels of the FBMC based identifiers approximately $3 \mathrm{~dB}$ lower than in the case of OFDM based identifiers. In free space, this may increase the wake-up radio receiver range by approximately $40 \%$.

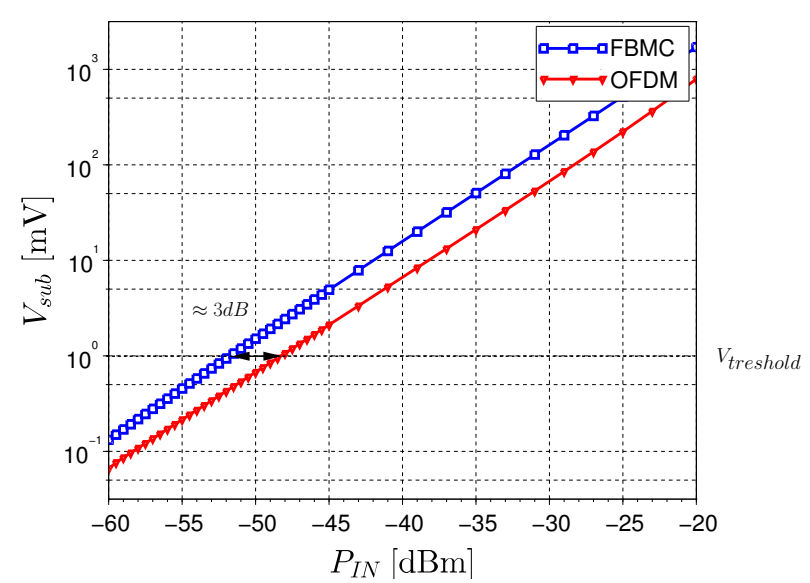

Fig. 3. The $V_{\text {sub }}$ subtractor's output when OFDM and FBMC signals are used

One of the important parameters of such kind of receiver is its latency, defined as the time lapse between the identifier reception and the output activation. Compared to the OFDM case, when using FBMC signals to generate identifiers, the latency is reduced particularly when low power level identifiers are received. This can be noticed on Figure 4 and can be explained by the higher efficiency of the rectifier circuits in the presence of higher PAPR signals. In this case, the $V_{D C 1}$ and $V_{D C 2}$ output voltages on the direct and complementary paths are higher than in the case of the OFDM and so, the threshold level is reached more rapidly.

Another advantage of using the FBMC signals to form the identifiers is the increasing immunity to the disparity of

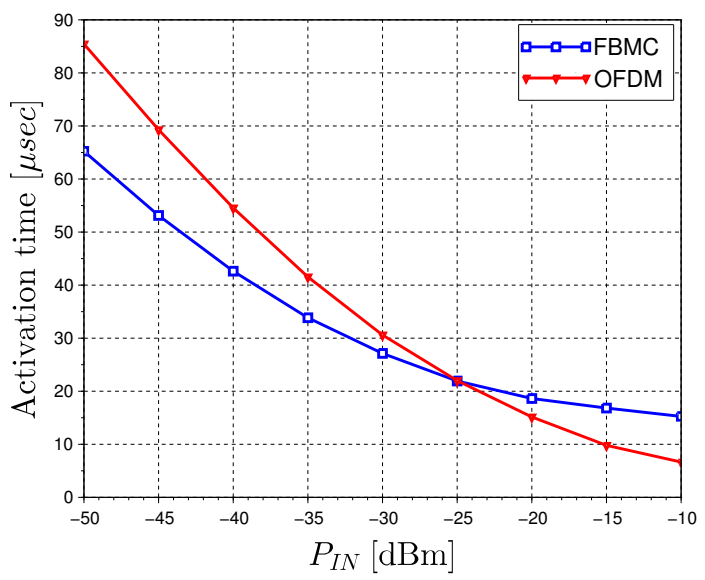

Fig. 4. The activation time for different power levels of the FBMC and OFDM received identifiers 




Fig. 5. The effect of the filters central frequency shifting on the WuRx robustness

the filters' central frequency. In order to test this increase of robustness, the direct and complementary path filters' central frequencies were shifted by $\varepsilon_{f}$. As can be noticed from Figure 5, in the case of FBMC identifiers, the allowed $\varepsilon_{f}$ error is about $3 \mathrm{MHz}$ and in the case of the OFDM identifier only 1.2 MHz. Here, the two identifiers (FBMC and OFDM), were generated with $-30 \mathrm{dBm}$ power level.

\section{CONCLUSION}

In this paper, the use of FBMC signals as frequency fingerprints for quasi-passive wake-up radio receivers was addressed. This kind of identifiers allows reducing the energy consumption of a wake-up radio receiver because they are eliminating the need of a microcontroller or of an analog correlator circuit. Because of their increased performances compared to the OFDM signals, the sensitivity, the latency and the robustness of the WuRx are improved. This is demonstrated through circuit-system co-simulations performed by using Keysight's ADS and SystemVue software. Future work will be devoted to validate experimentally the obtained results. Moreover, complementary efforts are undertaken in order to consider the propagation channel effect on the overall performances. Indeed, in complex environments, the selective fading may affect the shape of the received identifier and thus produce false wake-ups.

\section{ACKNOWLEDGMENT}

This work was supported by INSA Lyon's BQR (Bonus Qualité Recherche) grant Atchoum.

\section{REFERENCES}

[1] D. Evans, "The internet of things. how the next evolution of the internet is changing everything," CISCO, 2011.

[2] F. Boccardi, R. W. Heath, A. Lozano, T. L. Marzetta, and P. Popovski, "Five disruptive technology directions for 5G," IEEE Communications Magazine, vol. 52, no. 2, pp. 74-80, February 2014.
[3] F. Schaich and T. Wild, "Waveform contenders for 5G - 2014; OFDM vs. FBMC vs. UFMC," in 2014 6th International Symposium on Communications, Control and Signal Processing (ISCCSP), May 2014, pp. 457-460.

[4] E. T. Armas, D. Ramos-Valido, S. L. Khemchandani, and J. del Pino, "A $40.9 \mu \mathrm{W}$ high sensitivity wake-up radio for wireless sensor networks using uncertain-IF architecture," in 2015 Conference on Design of Circuits and Integrated Systems (DCIS), Nov 2015, pp. $1-6$.

[5] M. Magno, V. Jelicic, B. Srbinovski, V. Bilas, E. Popovici, and L. Benini, "Design, implementation, and performance evaluation of a flexible low-latency nanowatt wake-up radio receiver," IEEE Transactions on Industrial Informatics, vol. 12, no. 2, pp. 633-644, April 2016.

[6] H. Jiang, P. H. P. Wang, L. Gao, P. Sen, Y. H. Kim, G. M. Rebeiz, D. A. Hall, and P. P. Mercier, "A $4.5 \mathrm{nW}$ wake-up radio with -69 dBm sensitivity," in 2017 IEEE International Solid-State Circuits Conference (ISSCC), Feb 2017, pp. 416-417.

[7] F. Hutu, A. Khoumeri, G. Villemaud, and J.-M. Gorce, "A new wake-up radio architecture for wireless sensor networks," EURASIP Journal on Wireless Communications and Networking, vol. 2014, no. 1, pp. 1-10, 2014.

[8] F. Hutu, D. Kibloff, G. Villemaud, and J. M. Gorce, "Experimental validation of a wake-up radio architecture," in Proc. IEEE Radio and Wireless Symp. (RWS), Jan. 2016, pp. 155-158.

[9] A. Boaventura, D. Belo, R. Fernandes, A. Collado, A. Georgiadis, and N. B. Carvalho, "Boosting the efficiency: Unconventional waveform design for efficient wireless power transfer," IEEE Microwave Magazine, vol. 16, no. 3, pp. 87-96, April 2015.

[10] M. D. Prete, A. Costanzo, M. Magno, D. Masotti, and L. Benini, "Optimum excitations for a dual-band microwatt wake-up radio," IEEE Transactions on Microwave Theory and Techniques, vol. 64, no. 12, pp. 4731-4739, Dec 2016.

[11] M. L. Ku, Y. Han, H. Q. Lai, Y. Chen, and K. J. R. Liu, "Power waveforming: wireless power transfer beyond time reversal," IEEE Transactions on Signal Processing, vol. 64, no. 22, pp. 5819-5834, Nov 2016.

[12] J. B. . J. K. . H. Karl, "A survey of low-power transceivers and their applications," IEEE Circuits and Systems Magazine, vol. 15, pp. 6 - 17, 2015.

[13] L. Gu and J. A. Stancovic, "Radio-triggered wake-up for wireless sensor networks," Real-Time Systems, vol. 29, pp. 157 - 182, 2005.

[14] C. Hambeck, S. Mahlknecht, and T. Herndl, "A $2.4 \mu W$ wakeup receiver for wireless sensor nodes with $-71 \mathrm{dBm}$ sensitivity," in IEEE International Symposium on Circuits and Systems, May 2011.

[15] J. Liu, C. Li, L. Chen, Y. Xiao, J. Wang, H. Liao, and R. Huang, “An ultra-low power $400 \mathrm{MHz}$ OOK transceiver for medical implanted applications," in 2011 Proceedings of the ESSCIRC (ESSCIRC), sept. 2011, pp. $175-178$.

[16] M. Vidojkovic, X. Huang, P. Harpe, S. Rampu, C. Zhou, L. Huang, K. Imamura, B. Busze, F. Bouwens, M. Konijnenburg, J. Santana, A. Breeschoten, J. Huisken, G. Dolmans, and H. de Groot, "A $2.4 \mathrm{GHz}$ ULP OOK single-chip transceiver for healthcare applications," in 2011 IEEE International Solid-State Circuits Conference Digest of Technical Papers (ISSCC), feb. 2011, pp. $458-460$.

[17] R. Hamdi, A. Bounif, A. Desmarais, D. Deslandes, and F. Nabki, "A low-power OOK ultra-wideband receiver with power cycling," in New Circuits and Systems Conference (NEWCAS), 2011 IEEE 9th International, june 2011, pp. $430-433$.

[18] V. Rosello, J. Portilla, and T. Riesgo, "Ultra low power FPGAbased architecture for wake-up radio in wireless sensor networks," in IECON 2011 - 37th Annual Conference on IEEE Industrial Electronics Society, nov. 2011, pp. 3826 -3831.

[19] G. U. Gamm, M. Sippel, M. Kostic, and L. M. Reindl, "Low power wake-up receiver for wireless sensor nodes," in International Conference on Intelligent Sensors, Sensor Networks and Information Processing, December 2010

[20] A. Collado and A. Georgiadis, "Improving wireless power transmission efficiency using chaotic waveforms," in 2012 IEEE/MTT-S International Microwave Symposium Digest, June 2012, pp. 1-3. 